\title{
Online Tuning of Two Degrees of Freedom Fractional Order Control Loops
}

\author{
A. Ates, and C. Yeroglu
}

\begin{abstract}
This paper presents online tuning of Two Degrees of Freedom control loops with fractional order proportionalintegral-derivative controller. Since, simultaneous system objectives can be achieved by these types of control loops it can be used for challenging control problems. Thus, five various control loops are reconfigured with fractional order integral and derivative expressions for real time controller tuning problem. Seven parameters of the modified control loops are optimized via stochastic multi parameter divergence optimization algorithm. The optimization algorithm employs good performance for online tuning. The performance of the five various structures are compared using simulation model and real time experimental study on a prototype flight control simulator.
\end{abstract}

Index Terms-Control design, Closed loop systems, Position control, Design optimization, Stochastic processes.

\section{INTRODUCTION}

$\mathrm{O}$ NE Degree of Freedom (1DOF) control configuration generally unable to solve problems of achieving desired system response and sufficient feedback performance simultaneously. Two Degrees of Freedom (2DOF) control loop emerges as a necessary control tool to attain these two simultaneous system objectives. Research on 2DOF control structure reaches back to Horowitzh's study [1]. The major advantage of the 2DOF control structure is that the steady state error to the unit step change of the set-point variable and steady state error to the unit step disturbance become robustly zero [2,3]. Araki and Taguchi have presented important results about 2DOF structure with proportional-integral-derivative (PID) controller for tutorial purpose, including equivalent transformations and various explanations [4]. Many design studies of 2DOF control loop with PID (2DOF-PID) were already studied in the literature [5-7].

Thus, researches on control performance of 2DOF structure are still promising subject. In this respect, a fractional integral and differentiation may increase the possibility of fine tuning on controller parameters that yield better control performance on 2DOF structure [8-11]. Fractional order controllers are used with 2DOF in recent studies [12,13]. Being a keystone study on the fractional order controller researches, Podlubny has proposed Fractional Order PID (FOPID) controller in [14]. Then, many analytical design procedures were studied $[15,16]$.
On the other hand, several numerical tuning methods were also proposed for FOPID controllers tuning [17-19].

Nowadays, Stochastic Multi-parameter Divergence Optimization (SMDO) algorithm has been used as an effective numerical tuning method for FOPID controller tuning [20], $[21,22]$. The SMDO algorithm may provide effective results for 2DOF with FOPID (2DOF-FOPID) control loops.

In the proposed study, five different types of 2DOF structure, namely FeedBack (FB), Feed-Forward (FF), Component Separated (CS), Set-Point Filter type (SPF) Filter Preceded-Derivative (FPD) types in [3] are modified using FOPID controller. Then, five parameters of the FOPID controller and two parameters of 2DOF control structures were optimized simultaneously using SMDO algorithm for simulation model and real time experimental setup of Twin Rotor MIMO (Multi Input Multi Output) System (TRMS). Then the performances of each 2DOF-FOPID control loops are compared.

The TRMS experimental setup provides a nonlinear and complex helicopter flight control test platform to simulate complex mechanisms of aircrafts [23]. The adaptive control skill based on online controller tuning is required to maintain control performance under the variable conditions found in real-time flight applications. The novelty of the paper lies in introducing SMDO method for 2DOF-FOPID control loop optimization for real time flight control simulator.

Following sections provides two degree of freedom FOPID control structure, brief introduction to the TRMS and SMDO method, simulation and experimental results, respectively.

\section{2DOF CONTROL STRUCTURE}

2DOF control system in Fig.1 consists of serial compensator $C(s)$ and feedforward compensator $C_{f}(s)$. Let disturbance input be $P_{d}(s)$ and plant transfer function be $P(s)[2,3]$. The closed-loop transfer functions from set point variable $(r)$ to output $(y)$ and form disturbance input $(d)$ to $(y)$ are given, respectively as,

$$
G_{y r 2}=\frac{P(s)\left\{C(s)+C_{f}(s)\right\}}{1+P(s) C(s) H(s)}
$$

$$
G_{y d 2}=\frac{P_{d}(s)}{1+P(s) C(s) H(s)}
$$


It can be shown that the steady-state error to the unit step change of the set point variable $\varepsilon_{r, s t e p}$ and the steady-state error to unit step disturbance, $\varepsilon_{d, \text { step }}$ become robustly zero if conditions $\quad \lim _{s \rightarrow 0} C(s)=\infty, \lim _{s \rightarrow 0} \frac{C_{f}(s)}{C(s)}=0, \quad \lim _{s \rightarrow 0} H(s)=1$, $\lim _{s \rightarrow 0} P(s) \neq 0, \lim _{s \rightarrow 0}\left|\frac{P_{d}(s)}{P(s)}\right|<\infty$ are satisfied [2,3].

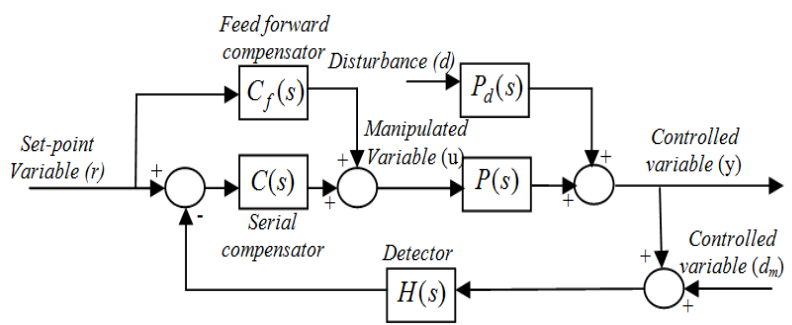

Fig. 1. 2DOF Control Structure

\section{2DOF FRACTIONAL CONTROL LOOP OPTIMIZATION}

In order to improve system performance, the system sensitivity and transmission function can be realized independently by 2DOF configuration [1]. The performance of the $2 \mathrm{DOF}$ control loop can be further improved by using fractional order controller for new control loop design.

Some recent studies present the advantage of the following FOPID controller [14] over conventional PID in control system design,

$$
C(s)=P I^{\lambda} D^{\mu}=k_{p}+\frac{k_{i}}{s^{\lambda}}+k_{d} s^{\mu}
$$

FOPID controller in Eq. 3 can be used to improve the performance of $2 \mathrm{DOF}$ control structure. Motivation of the present study is to improve the performance of $2 \mathrm{DOF}$ configuration with FOPID via a recent optimization algorithm. In this study, the fundamental $2 \mathrm{DOF}$ structures in $[1,3,12]$ have been modified with FOPID to obtain effective control loops as in Figs. 2-6. Fig. 2 shows that FF type 2DOF-FOPID control loop can be obtained by adding feed forward path to the output of the FOPID control signal.

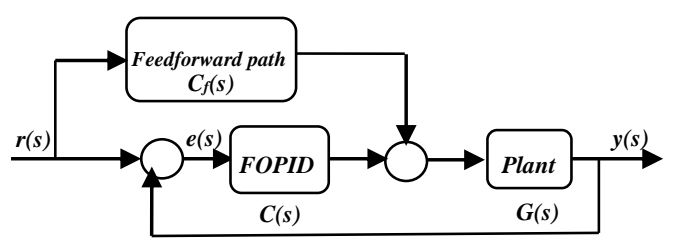

Fig. 2. FF type 2DOF-FOPID Control Structure

One can compute the following transfer function for the feedforward path using proportional and differential parameters of the FOPID controller.

$$
C_{f}(s)=-k_{p} \alpha-\beta k_{d} s^{\mu}
$$

where, $\alpha$ is the coefficient of the proportional gain $(0<\alpha<1), \quad \beta$ is coefficient of the differentiation gain $(1<\beta<2)$. Fig. 3 gives FB type 2DOF-FOPID control loop, where $C_{b}$ is called feedback compensator. Following transfer function for the feedback path can be obtained using proportional and fractional integro-differential parameters of the FOPID controller,

$$
C^{\prime}(s)=(1-\alpha) k_{p}+\frac{k_{i}}{s^{\lambda}}+(1-\beta) k_{d} s^{\mu}
$$

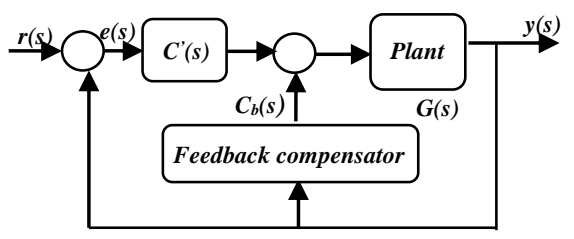

Fig. 3. FB type 2DOF-FOPID Control Structure

The transfer function for the feedback compensator $C_{b}$ is also computed using proportional and differential parameters as follows,

$$
C_{b}(s)=k_{p} \alpha+k_{d} \beta s^{\mu}
$$

CS, SPF and FPD type 2DOF PID control loops in [3] is modified using FOPID controller as given in Figs. 4, 5 and 6, respectively. The transfer functions $F_{S P F}(s)$ in Fig. 5 and $F_{F P D}(s)$ in Fig. 6 can be obtained as in Eqs. 7 and 8.

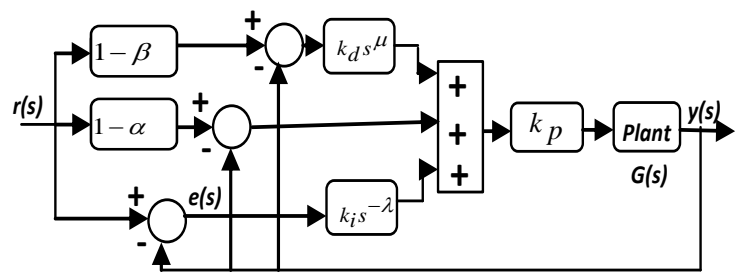

Fig. 4. CS type 2DOF-FOPID Control Structure

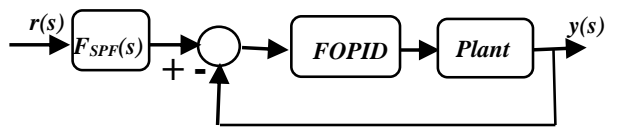

Fig. 5. SPF type 2DOF-FOPID Control Structure

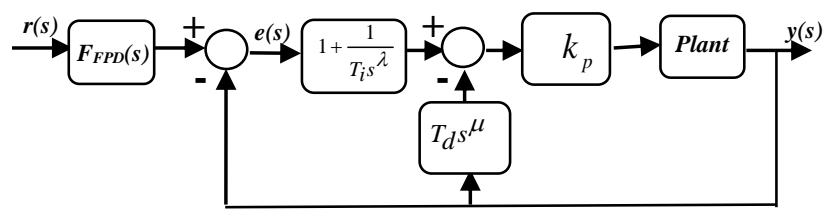

Fig. 6. FPD type 2DOF-FOPID Control Structure

$$
\begin{gathered}
F_{S P F}(s)=\frac{1+(1-\alpha) T_{i} s^{\lambda}+(1-\beta) T_{i} T_{d} s^{\mu}}{1+T_{i} s^{\lambda}+T_{i} T_{d} s^{\mu}} \\
F_{F P D}(s)=\frac{1+(1-\alpha) T_{i} s^{\lambda}+(1-\beta) T_{i} T_{d} s^{\mu}}{1+T_{i} s^{\lambda}}
\end{gathered}
$$


Totally, seven parameters of the FF, FB, CS, SPF and FPD type 2DOF-FOPID control loops should be optimized to provide best control performance. However, SMDO emerges as an effective optimization tool to obtain optimum parameters for the proposed control loops in Figs. 2-6. Practical implementation of FOPID controller has some difficulties for realization of the fractional-order derivative and integrator terms. Several methods are used for realization of the FOPID controller in literature [24]. Valerio proposed a non-integer toolbox for realization of the FOPID controller by combining following three approximation methods [25]; i) The Crone, ii) Carlson's and iii) Matsuda's methods can be summarized as following $[24,26]$;

$$
\begin{gathered}
C(s)=\tilde{k} \prod_{n=1}^{N} \frac{1+s / w_{z n}}{1+s / w_{p n}} \\
C_{n}(s)=C_{n-1}(s) \frac{\alpha-(1) C_{n-1}{ }^{\alpha}(s)+(\alpha+1) g(s)}{(\alpha+1) C^{\alpha}{ }_{n-1}+(\alpha-1) g(s)} \\
C(s)=a_{0}+\frac{s-s_{0}}{a_{1}+} \frac{s-s_{1}}{a_{2}+} \frac{s-s_{2}}{a_{3}+}
\end{gathered}
$$

Thus the Valerio's toolbox benefits from these three methods to implement approximate solutions for FOPID. In this study, we used Valerio's toolbox to implement FOPID in our experimental setup.

Optimal performance of 2DOF-FOPID control loops in Figs. 2-6 can be reached using SMDO algorithm, which can optimize two parameters of the 2DOF structure and five parameters of FOPID controller simultaneously. SMDO algorithm in [20-22] is modified for 2DOF-FOPID control loop.

Let us denote coefficients of the 2DOF-FOPID control loop at time instance $t=n h$ by the vector $X^{n}=\left[k_{p}^{n} k_{i}^{n} k_{d}^{n} \lambda^{n} \mu^{n} \alpha^{n} \beta^{n}\right]$ in a parameter space $X$. Here, parameters $h$ represent the sampling period of discrete state sampling, and $n=\{1,2,3, \ldots\}$ is the discrete time increment. In order to adapt a 2DOF-FOPID control loop to the system, $X^{n}$ turns into $X^{m}$ to satisfy the following adaptation condition,

$$
E\left(X^{m}\right)-E\left(X^{n}\right)<0
$$

where $E$ is a positive real-valued error function that is used to evaluate the performance of the parameter optimization. SMDO uses consecutive set and trial tasks for optimization of parameter vectors. The objective of SMDO is to transform initial $X^{0}$ parameter vector to the optimal vector $X_{o p t}$. The optimization ends when the following equation is satisfied,

$$
E\left(X_{o p t}\right)=\min _{X^{n} \in X_{s}}\left\{E\left(X^{n}\right)\right\}
$$

The pseudo code of the modified SMDO algorithm in Fig. 7 is given to optimize seven parameters of 2DOF-FOPID control loop. Main step of the algorithm is to generate a divergence vector. Let a divergence vector be,

$$
\Delta X^{n}=\varphi_{v}^{n}\left[\Delta k_{p}^{n} \Delta k_{i}^{n} \Delta k_{d}^{n} \Delta \lambda^{n} \Delta \mu^{n} \Delta \alpha^{n} \Delta \beta^{n}\right]
$$

Forward and backward steps can be obtained according to divergence vector. The modified SMDO algorithm can search optimum solution in parameter space.

Components of forward divergence and arithmetic expression to test convenient divergence can be written respectively as,

$$
\begin{gathered}
X^{n+1}=X^{n}+\varphi_{v}^{n}\left[\Delta k_{p}^{n} \Delta k_{i}^{n} \Delta k_{d}^{n} \Delta \lambda^{n} \Delta \mu^{n} \Delta \alpha^{n} \Delta \beta^{n}\right] \\
E\left(X^{n}+\Delta X^{n}\right)-E\left(X^{n}\right)<0
\end{gathered}
$$

Similarly, components of backward divergence and related test expressions can be written respectively as,

$$
\begin{gathered}
X^{n+1}=X^{n}-\varphi_{\nu}^{n}\left[\Delta k_{p}^{n} \Delta k_{i}^{n} \Delta k_{d}^{n} \Delta \lambda^{n} \Delta \mu^{n} \Delta \alpha^{n} \Delta \beta^{n}\right] \\
E\left(X^{n}-\Delta X^{n}\right)-E\left(X^{n}\right)<0
\end{gathered}
$$

where, $\varphi_{v}$ is the divergence rate vector.

$$
\begin{aligned}
& \text { Start } \\
& \text { Parameter Initialization } \\
& \text { Starting point: } \\
& \text { If } E\left(X^{n}\right)>E_{\min } \\
& \text { Adaptation;Goto Adapted } \\
& v=v+1 \quad \text { if } v>7 \\
& v=0 \\
& \text { end } \quad \text { Compose } \Delta X^{n} \\
& \text { Forward test: } \\
& \quad \text { if } E\left(X^{n}+\Delta X^{n}\right)-E\left(X^{n}\right)<0 \\
& \quad X^{n+1}=X^{n}+\Delta X^{n} \\
& \quad \text { Go to Starting Point } \\
& \text { end } \\
& \quad \text { Backward test: } \\
& \text { if } E\left(X^{n}+\Delta X^{n}\right)-E\left(X^{n}\right)<0 \\
& \quad X^{n+1}=X^{n}-\Delta X^{n} \\
& \quad \text { Go to Starting Point } \\
& \text { end } \\
& \text { Go to Starting Point } \\
& \text { Adopted } \\
& \text { end } \quad \\
& \text { end } \quad
\end{aligned}
$$

Fig. 7. Draft code of the SMDO algorithm for tuning 2DOF-FOPID control structure. 


\section{2DOF-FOPID CONTROL LOOP FOR TRMS}

This section presents five types of 2DOF-FOPID control loops for simulation and real time models of a TRMS. Performance of the control loops are tested for simulation model, than applied to the real time experimental setup.

\section{A. Twin Rotor MIMO Systems (TRMS)}

The TRMS in Fig. 8 is widely used for flight control experiments. TRMS is a two-rotor system that maneuvers by vertical and horizontal rotors. The TRMS control problem is characterized a highly nonlinear multi-input multi-output problem [27-29]. Fig. 8 shows the experimental system developed for 2DOF-FOPID control loop tuning. The vertical and horizontal angle controls were tested in our experiment. The objective of this study is to make the TRMS move accurately and quickly to the desired attitudes and directions, by controlling the horizontal and vertical angles.

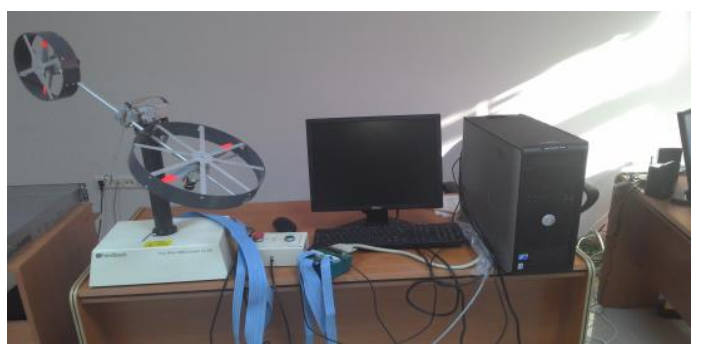

Fig. 8. TRMS Experimental Setup

In this paper, a mathematical model of TRMS main pitch rotor was obtained for simulation studies. Firstly multisinusoidal signal was applied to TRMS main pitch rotor to obtain relation vector that is between TRMS output and multisinusoidal signal. The system run during $100 \mathrm{sec}$ with $0.1 \mathrm{sec}$ sampling time. Then the data is transferred to MATLAB "system identification toolbox". Second order mathematical model in Eq. 19 for TRMS main pitch rotor was obtained according to "output error", linear parametric model structure with MATLAB system identification toolbox. This mathematical model is used for TRMS simulation setup.

$$
G_{\text {TRMS }}(s) \frac{0.06725+1.359}{s^{2}+0.7906 s+3.666}
$$

Five different 2DOF-FOPID control loops, namely FF, FB, CS, SPF and FPD types, are used with second order mathematical model of TRMS main rotor in Eq. 19 for the simulation study. The control performances of these control structures are compared to point out the more suitable 2DOFFOPID control loop. Simulation and experimental result are obtained according to TRMS optimization setup in Fig. 9. Real time experimental study takes initial values from the results of simulation.

\section{B. Simulation Study}

The second order mathematical model in Eq. 19 is used to represent TRMS main rotor. FF, FB, CS, SPF and FPD type 2DOF-FOPID control loops in Figs. 2-6 are used in simulation setup in Fig. 9 respectively. The "integer" block in [25] is used to realize fractional order integral and derivative. The parameters of control loops are obtained using SMDO algorithm in Fig. 7. The best cost function for optimum values is obtained during the simulation study by operating the simulation for 15 seconds using ode45 Matlab Simulink Solver with variable-steps [30], for all iterations. The cost function for the SMDO algorithm is defined as,

$$
J(\theta)=\min \frac{1}{N} \sum E^{2}
$$

where $E$ in real valued error function (cost function). Then, seven optimum parameters of FF, FB, CS, SPF and FPD type 2DOF-FOPID control loops are obtained as given in Table 1. Fig. 10 demonstrates the step responses of the system for the proposed FF, FB, CS, SPF and FPD type 2DOF-FOPID control loops in Table 1. The best step response is obtained for FB type 2DOF-FOPID control loop. Figs. 11 and 12 present the behavior of the cost function and parameters variation for the FB type 2DOF-FOPID, respectively that demonstrates the effectiveness of the optimization. Fig. 13 compares values of cost functions for control loops. The best cost function is also obtained for FB type 2DOF-FOPID control loop.

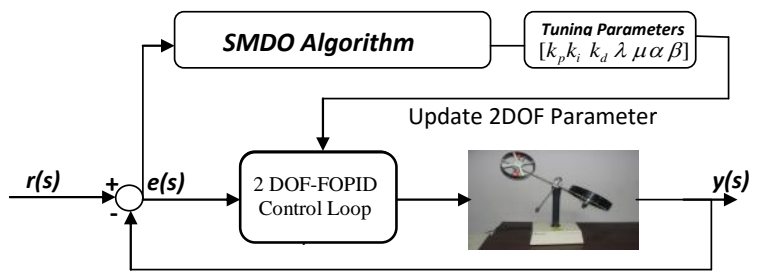

Fig. 9. Optimization process for simulation and experimental setup.

TABLE 1: PARAMETERS OF THE FIVE VARIOUS 2DOF-FOPID CONTROL STRUCTURES THAT ARE GENERATED BY THE SMDO ALGORITHM FOR SIMULATION STUDY

\begin{tabular}{|l|c|c|c|c|l|}
\hline & FF & FB & CS & SPF & \multicolumn{1}{|c|}{ FPD } \\
\hline$k_{p}$ & 7.9888 & 10.8318 & 15.9728 & 79.8708 & 63.0741 \\
\hline$k_{i}$ & 3.9993 & 3.3221 & 3.9337 & 31.8713 & 48.3935 \\
\hline$k_{d}$ & 13.3757 & 12.5241 & 12.2003 & 24.4112 & 33.1517 \\
\hline$\lambda$ & 0.9698 & 0.9374 & 0.5690 & 0.9334 & 0.5323 \\
\hline$\mu$ & 1.2454 & 1.2845 & 0.7529 & 0.9722 & 0.5953 \\
\hline$\alpha$ & 0.3169 & 0.3077 & 0.1356 & 2.1217 & 0.9906 \\
\hline$\beta$ & 0.5422 & 0.1300 & 0.6106 & 0.7555 & 0.3057 \\
\hline e) & 0.0037 & $5.03 * 10^{-5}$ & 0.0119 & 0.0082 & $1.62^{*} 10^{-4}$ \\
\hline
\end{tabular}




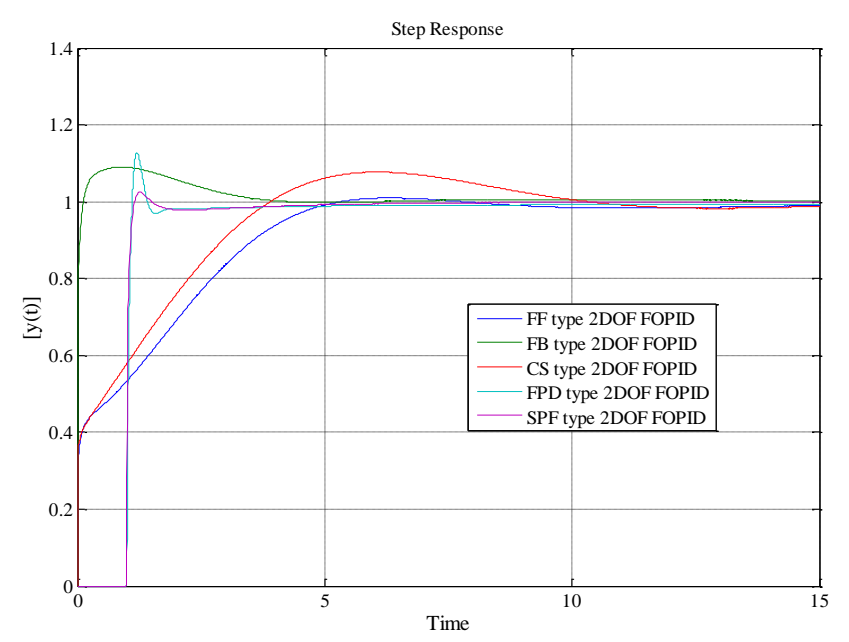

Fig. 10. Step Responses of FF, FB, CS, SPF, FPD types control loops.

\section{Experimental Study}

This section tests the proposed SMDO method experimentally on TRMS system in the optimization setup in Fig. 9. SMDO algorithm in Fig. 7 is employed for this system. "Advantech PCI 1711" interface card is equipped to control TRMS and data acquisition. The real time experimental study is carried on experimental setup in Fig. 9 for FF, FB, CS, SPF and FPD types 2DOF-FOPID control loops respectively. Results of the optimization algorithm are provided in Table 2. Fig. 14 shows the step responses of SMDO for FF, FB, CS, SPF and FPD type 2DOF-FOPID control loops. The best performance is obtained for FF type 2DOF-FOPID control loop in real time application. Fig. 15 provide the variation the cost function during the optimization process.

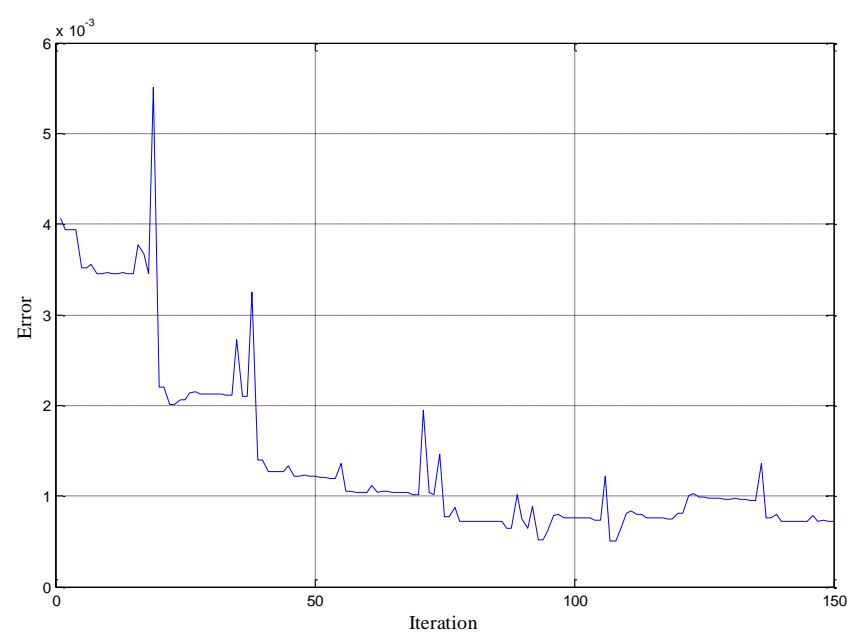

Fig. 11. Variation of the cost function for FB type 2DOF-FOPID during the optimization process.

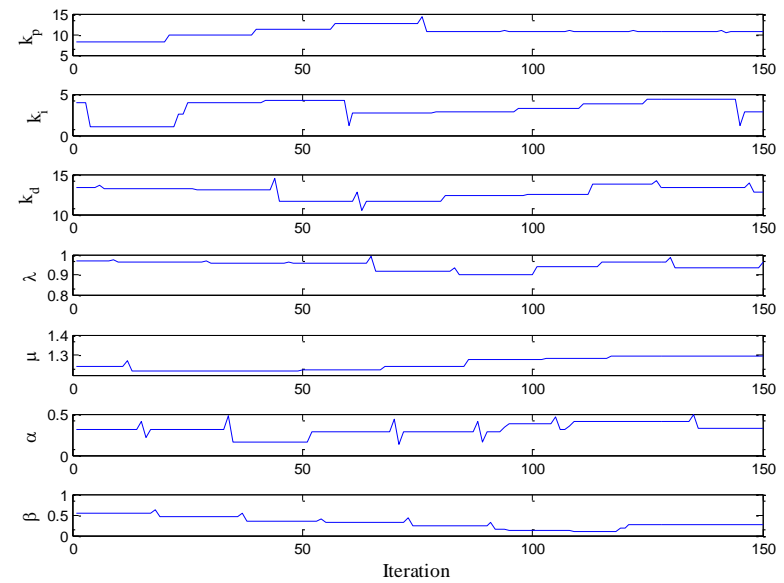

Fig. 12. Parameter variation of FB type 2DOF-FOPID during the optimization process.

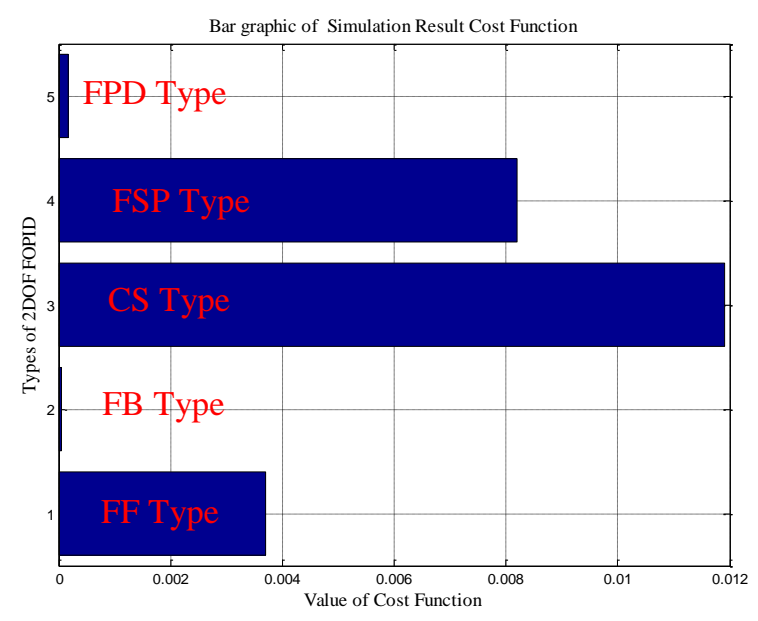

Fig. 13. Simulation result of cost function value (E).

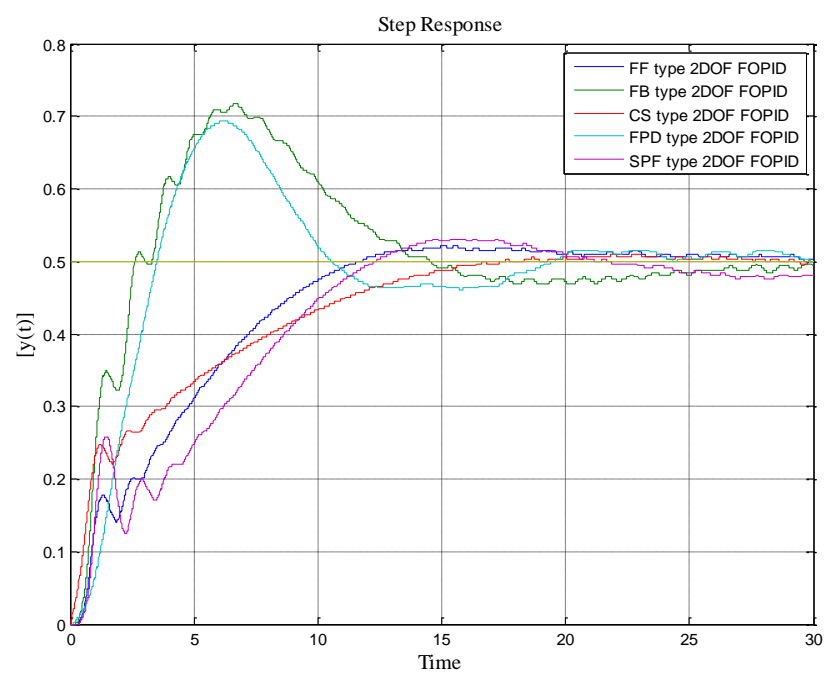

Fig. 14. Comparison of five various 2DOF-FOPID control structures. 


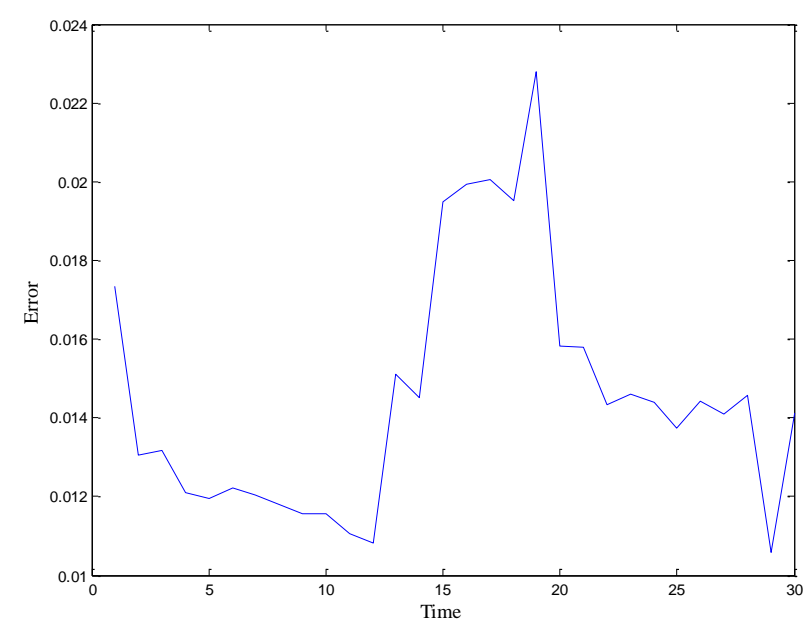

Fig. 15. Variation of the cost function for FF type 2DOF-FOPID during the optimization process.

TABLE 2: PARAMETERS OF THE FIVE VARIOUS 2DOF-FOPID CONTROL STRUCTURES THAT ARE GENERATED BY THE SMDO ALGORITHM FOR EXPERIMENTAL STUDY.

\begin{tabular}{|l|l|l|l|l|l|}
\hline & FF & FB & CS & SPF & FPD \\
\hline$k_{p}$ & 5.4582 & 8.9189 & 0.9593 & 1.8147 & 0.9514 \\
\hline$k_{i}$ & 1.3239 & 1.7707 & 1.6041 & 0.8000 & 0.8714 \\
\hline$k_{d}$ & 18.8850 & 18.0714 & 16.6405 & 13.2539 & 3.1982 \\
\hline$\lambda$ & 1.1456 & 1.0646 & 1.1244 & 1.0464 & 1.0322 \\
\hline$\mu$ & 1.1273 & 1.0505 & 1.0938 & 1.0716 & 1.1513 \\
\hline$\alpha$ & 0.8303 & 0.8662 & 0.5292 & 0.5195 & 0.8168 \\
\hline$\beta$ & 5.0000 & 5.0000 & 4.9290 & 4.9721 & 4.9040 \\
\hline $\mathbf{e})$ & 0.0106 & 0.0232 & 0.0132 & 0.1196 & 0.0125 \\
\hline
\end{tabular}

\section{CONCLUSION}

This paper presents the optimization of FF, FB, CS, SPF and FPD types 2DOF-FOPID control loops for simulation and real time TRMS experimental setup using SMDO algorithm. Generally, 2DOF controller design procedures were given in two steps; First step is to optimize controller and second step is to optimize 2DOF structure according to fixed controller value. But, SMDO algorithm gives opportunity to optimize the fractional order controller parameters and 2DOF structure parameters simultaneously to achieve better control performance. This study illustrates the advantage of 2DOFFOPID control structures and performance of SMDO algorithm.

\section{REFERENCES}

[1] I. M. Horowitz, Synthesis of Feedback Systems, Academic Press, New York, The University of Michigan, pp.1-726, 1963.

[2] M. Araki, "PID control system with reference feedforward (PID-FF control systems)", Proc 23rd SICE (Society of Instrument and Control Engineers) Annual Conference, pp.31-32, 1984.

[3] M. Araki, H. Taguchi, "Two-degree-of-freedom PID controllers", International Journal of Control Automation and Systems, Vol.1, pp. 401-411, 2003.

[4] T. Nagashio, T. Kida, Y. Hamada, T. Ohtani, "Robust Two-Degrees-ofFreedom Attitude Controller Design and Flight Test Result for Engineering Test Satellite-VIII Spacecraft”, Control Systems Technology, Vol. 22, No.1, pp.157-168, 2014.
[5] M. Ajmeri, A. Ali, "Two degree of freedom control scheme for unstable processes with small time delay", ISA Transactions, Vol.56, pp.308-326, 2015.

[6] B. B. Ghosh, B. K. Sarkar, R. Saha, "Realtime performance analysis of different combinations of fuzzy-PID and bias controllers for a two degree of freedom electro hydraulic parallel manipulator", Robotics and Computer-Integrated Manufacturing, Vol. 34, pp. 62-69, 2014.

[7] G. H. Choi, K. Park, J. H. Jung, "An optimal $\mathrm{H}_{2}$ decoupling design for non-square plant systems based on the two-degree-of-freedom standard model", International Journal of Control, Automation and Systems, Vol.7, No.2, pp.193-202, 2009.

[8] R. E. Gutierrez, J. M. Rosario, J. A. T. Machado, "Fractional Order Calculus: Basic Concepts and Engineering Applications", Mathematical Problems in engineering, Hindawi Publishing Corporation, Vol.2010, pp.1-9, 2010.

[9] I. Podlubny, Fractional Differential Equations, Mathematics in Science and Engineering, Academic Press, USA, 1999.

[10] B. Ross, Fractional Calculus and its Applications, Springer, Verlag, Berlin, New York, 1975.

[11] F. Merrikh-Bayat, N. Mirebrahimi, M. R. Khalili, "Discrete-time fractional-order PID controller: Definition, tuning, digital realization and some applications", International Journal of Control Automation and Systems, Vol.13, No.1, 81-90, 2015.

[12] G. Feng, Z. Xiao-ping, "Research on fractional order two-degrees-offreedom flight control technology of unmanned air vehicle", Computer Science and Information Processing, pp. 807-812, 2012.

[13] S. Debbarma, L. C. Saikia, N. Sinha, "Automatic generation control using two degree of freedom fractional order PID controller", International Journal of Electrical Power \& Energy Systems, Vol.58, pp. 120-129, 2014.

[14] I. Podlubny, "Fractional order systems and $\mathrm{PI}^{\lambda} \mathrm{D}^{\mu}$ controller", Proc. IEEE Trans. Automatic Control, Vol. 44, pp. 208-214, 1999.

[15] C. Yeroglu, N. Tan, "Note on fractional-order proportional-integraldifferential controller design", IET Control Theory and Applications, Vol. 5, No.17, pp.1978-1989, 2011.

[16] C. Yeroglu, N. Tan, "Classical controller design techniques for fractional order case", ISA Transactions, Vol.50, No.3, pp.461-472, 2011.

[17] R. El-Khazali, "Fractional-order controller design", Computers \& Mathematics with Applications, Vol. 66, No.5, pp.639-646, 2013.

[18] L. H. Sheng, Y. Luo, Y. Q. Chen, "A fractional order proportional and derivative (FOPD) motion controller: tuning rule and experiments", Control Systems Technology, Vol.18, No.2, pp. 516-520, 2010.

[19] A. Ates, C. Yeroglu, "Tabu Search Algorithm for Fractional Order PID via Non-linear Multi Objective Function", International Conference on Fractional Differentiation and Its Applications, Italy, 2014.

[20] C. Yeroglu, A. Ates, "A stochastic multi-parameters divergence method for online auto-tuning of fractional order PID controllers", Journal of the Frankline Institue, Vol.351, No.5, pp.2411-2429, 2014.

[21] B. B. Alagoz, A. Ates, C. Yeroglu, "Auto-tuning of PID controller according to fractional order reference model approximation for DC rotor control", Journal of the Mechatronics, Vol.23. No.7, pp.789-797, 2013.

[22] A. Ates C. Yeroglu, B. B. Alagoz, B. Senol, "Tuning of Fractional Order PID with Master Slave Stochastic Multi-Parameter Divergence Optimization Method", International Conference on Fractional Differentiation and Its Applications, Italy, 2014.

[23] D. Song, J. Han, G. Liu, "Active Model-Based Predictive Control and Experimental Investigation on Unmanned Helicopters in Full Flight Envelope", IEEE Transactions on Control System Technology, Vol.99, pp. 1-8, 2012.

[24] A. Oustaloup, La commande CRONE: commanderobusted'ordre non entire, Hermès, Paris, 1991.

[25] D.Valerio, Ninteger v. 2.3 Fractional Control Toolbox for MATLAB, 2005. [Online]. Available: http://web.ist.utl.pt/ duarte.vale

[26] K. Matsuda, H. Fujii, " $\mathrm{H}_{\infty}-$ optimized wave-absorbing control: analytical and experimental results", Journal of Guidance Control and Dynamics, Vol.16, No.6, pp.1146-1153, 1993.

[27] S. F. Ahammad, S. Purwar, "A nonlinear state observer design for 2-dof twin rotor system using neural networks", Advances in Computing, International Conference on Control, \& Telecommunication Technologies, Trivandrum, Kerala, India, 2009. 
[28] C. L. Shih, M. L. Chen, J. Y. Wang, "Mathematical Model and Set-point Stabilizing Controller Design of a Twin Rotor MIMO System", Asian Journal of Control, Vol.10, No.1, pp.107-114, 2008.

[29] J. J. Gau, W. K. Liu, C. Y. Tsai, "Intelligent control scheme for twin rotor MIMO system", IEEE International Conference on Mechatronics, Taipei, Taiwan, 2005.

[30] C. Xie, A. Mark, "Turnquist Lane-based evacuation network optimization: An integrated Lagrangian relaxation and tabu search approach", Transportation Research-Elseiver, Vol.19, No.1, pp.40-63, 2011.

\section{BIOGRAPHIES}

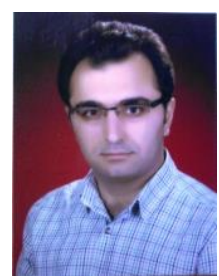

ABDULLAH ATES received his B. Sc. Degree in Electrical and Electronics Engineering from Erciyes University in 2011. He received his master degree in Computer Engineering from Inonu University in 2013. His research interests include optimization algorithm, analytical and numerical optimization method, fractional order control systems, fractional order calculus and its application, linear and nonlinear optimization algorithm and flight control.

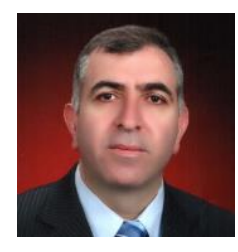

CELALEDDIN YEROĞLU received his B.Sc. degree in Electrical and Electronics Engineering from Hacettepe University in 1990 . He received his first Ph.D. degree in Computer Engineering from Trakya University in 2000 and his second Ph.D. degree in Electrical and Electronics Engineering from Inonu University in 2011. His research interests include fractional order control systems, robust control, nonlinear control, modeling and simulation, optimization algorithm, flight control. 